\title{
Effect of L-asparagine as dopant on the growth and characteristics of ammonium tetroxalate dihydrate single crystal
}

\author{
Eunice Jerusha ${ }^{1,2}$, S. Shahil KirupaVATHY ${ }^{3, *}$ \\ ${ }^{1}$ Department of Physics, R.M.D. Engineering College, Kavaraipettai 601206, India \\ ${ }^{2}$ Anna University Chennai, Guindy, Chennai 600 025, India \\ ${ }^{3}$ Department of Physics, Velammal Engineering College, Chennai 600066, India
}

\begin{abstract}
Single crystals of L-asparagine doped ammonium tetroxalate dihydrate were grown using slow evaporation solution growth technique with deionized water as a solvent. The shift and intensity of the peaks in the single crystal X-ray diffraction and Fourier transform infrared analyses confirmed the inclusion of L-asparagine in the ammonium tetroxalate dihydrate crystal structure. The optical transmission characteristics viz. optical band gap, optical conductivity, etc., were determined. Thermal studies revealed the occurrence of anomalies at $135.3^{\circ} \mathrm{C}$ and $221.7{ }^{\circ} \mathrm{C}$ leading to the possibility of phase transitions and thereby, ferroelectric behavior. Vickers microhardness studies enabled determination of various microhardness parameters. Dielectric behavior was analyzed by varying the temperature and frequency. Anomalies were observed at $135{ }^{\circ} \mathrm{C}$ and $221{ }^{\circ} \mathrm{C}$ suggesting the existence of ferroelectric nature of the compound. Self-defocusing effect was observed. The polarization-electric field hysteresis loops showed a pinched effect due to defects induced by inclusion of dopant. The crystals were characterized by single crystal XRD, FT-IR, DRS, UV-Vis-NIR, Vickers microhardness test as well as thermal and dielectric techniques. In brief, L-asparagine as a dopant in ammonium tetroxalate dihydrate altered various physical properties of the crystals. They were highly transparent in the visible region with a wider optical band gap, softer material than the parent, higher phase transition temperature of $135^{\circ} \mathrm{C}$, negative nonlinearity and self-defocusing ability.
\end{abstract}

Keywords: Z-scan; dielectric properties; thermal characteristics; organic crystal

\section{Introduction}

Ferroelectricity is a characteristic of certain materials to exhibit polarization due to an applied electric field. Such materials have potential versatile technical applications in ferroelectric random access memories (FeRAM), ferroelectric field effect transistors (FeFET), infrared (IR) detectors and tunable capacitors [1, 2]. Most of these crystals tend to depolarize with time, hence an efficient way to maintain the polarized nature is by the introduction of optically active molecules as dopants into the crystal structure. Due to the vast number of available organic compounds and the well-established methodology of organic synthesis which allows systematic structural modification of molecules, organic materials show

*E-mail: shahilkirupavathy@yahoo.co.in great promise for complementing or replacing currently employed inorganic substances in a variety of situations [3].

Specific properties of a material determine the effectiveness and efficiency of the device in which it is used. Amino compounds are of immense interest due to their physical characteristics. Organic electronics-based applications are very effective due to the flexibility and non-toxicity of organic molecules. Materials science has, therefore, a new challenge with the link between organic and ferroelectric molecules which is currently under study.

An addition of organic, semi-organic or inorganic impurities to a crystal leads to the modification of its structural, optical, mechanical and dielectric properties. Thus, the intake of the impurity species into a crystal has important implications in industrial crystallization. 
Ammonium tetroxalate dihydrate (ATOXAL) is a uniaxial semi-organic crystal, crystallizing in the triclinic system [4]. Further, detailed characterization of ATOXAL crystals revealed their ferroelectric nature [5]. In the present study, attempts were made to grow L-asparagine monohydrate (L-asp) doped ATOXAL crystals and investigate the influence of L-asp on various properties of the ATOXAL crystal. The chosen dopant L-asp is an important amino acid among biological substances since it plays a major role in the metabolic control of certain cell functions in nerves and brain tissues and also as a nitrogen reserve source in many plants. Chiral carbon atoms in the amino acid on doping into the crystal lattice of ATOXAL lead to the alteration of properties, favoring the crystal's non-centrosymmetric behavior and the hardness due to the zwitter ions. The variation in the growth and physical properties of ATOXAL on the usage of L-asp as a dopant are reported in detail.

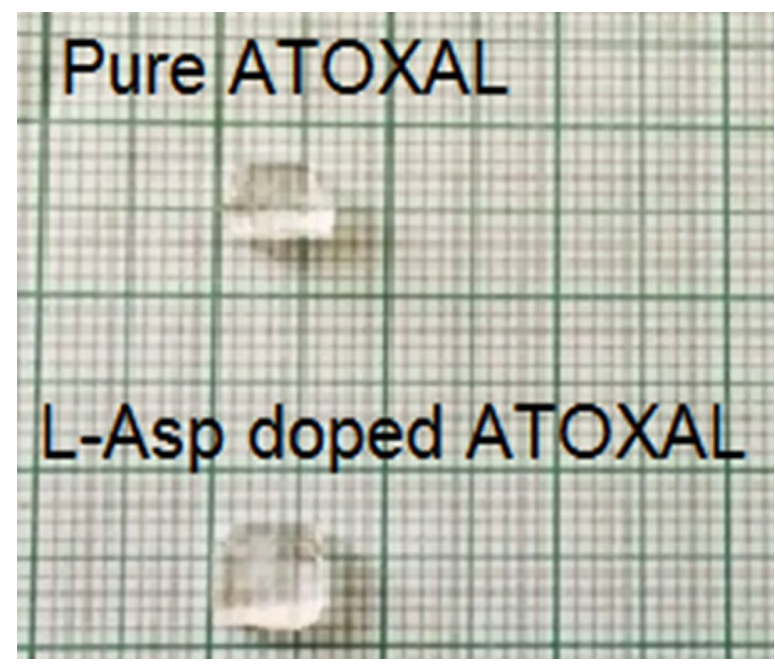

Fig. 1. As-grown pure and L-asp doped ATOXAL crystals.

Solution growth techniques are mainly applied for growing good quality single crystals. Low thermal stability of many examined compounds makes exploratory solution growth as the first technique to be considered. ATOXAL was prepared by mixing equimolar portions of ammonium oxalate (Merck, AR grade, $99 \%$ ) and oxalic acid (Loba, AR grade, $99 \%$ ) in deionized water. The solution was stirred at room temperature for about $6 \mathrm{~h}$ to get
Table 1. Comparison of crystallographic data of pure and L-asp doped ATOXAL.

\begin{tabular}{ccc}
\hline $\begin{array}{c}\text { Unit cell } \\
\text { parameters }\end{array}$ & $\begin{array}{c}\text { Pure } \\
\text { ATOXAL }\end{array}$ & $\begin{array}{c}\text { L-asp doped } \\
\text { ATOXAL }\end{array}$ \\
\hline \hline $\mathrm{a}[\AA]$ & 6.35 & $6.383( \pm 0.008)$ \\
$\mathrm{b}[\AA]$ & 7.25 & $7.271( \pm 0.010)$ \\
$\mathrm{c}[\AA]$ & 10.58 & $10.616( \pm 0.013)$ \\
$\alpha\left[^{\circ}\right]$ & 94.17 & $94.38( \pm 0.03)$ \\
$\beta\left[^{\circ}\right]$ & 100.12 & $100.12( \pm 0.05)$ \\
$\gamma\left[{ }^{\circ}\right]$ & 97.86 & $97.77( \pm 0.03)$ \\
$\mathrm{V}\left[\AA^{3}\right]$ & 472.3 & $478.1( \pm 0.18)$ \\
Crystal system & triclinic & triclinic \\
\hline
\end{tabular}

a homogeneous supersaturated solution. 10 at. of L-asp was added to the solution and agitated by continuous stirring for another $6 \mathrm{~h}$ period at room temperature. The obtained clear solution was filtered into clean beakers and kept for slow evaporation in a constant temperature bath. Colorless, transparent crystals were harvested after a period of 21 days. The photographs of the as-grown pure and L-asp doped ATOXAL crystals are shown in Fig. 1.

\section{Results and discussion}

\subsection{Solubility measurement}

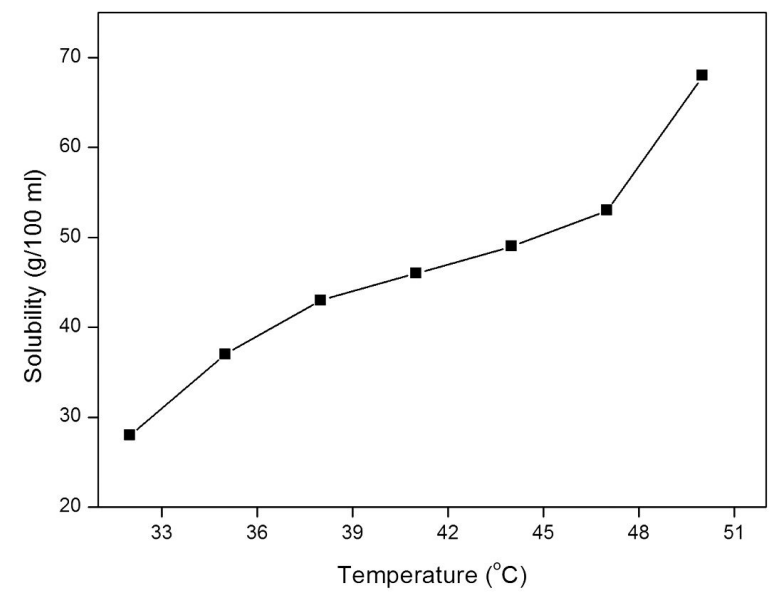

Fig. 2. Solubility curve of L-asp doped ATOXAL.

Solubility study of L-asp doped ATOXAL in water was performed in the temperature range 
between $30{ }^{\circ} \mathrm{C}$ and $55{ }^{\circ} \mathrm{C}$. The temperature was maintained above the chosen constant temperature and the solution was continuously stirred using a motorized magnetic stirrer to ensure homogeneous temperature and concentration throughout the volume of the solution. Gravimetric analysis was performed to test the solubility of the saturated solution. $100 \mathrm{~mL}$ of the solution was weighed and evaporated in an oven at a constant temperature. This process was repeated at different temperatures and the solubility temperature was found to be $42{ }^{\circ} \mathrm{C}$. The plot shown in Fig. 2 reveals that the solubility of L-asp doped ATOXAL increases linearly with temperature, exhibiting a high solubility gradient and positive temperature coefficient, which reveals the fact that slow evaporation technique is an appropriate method to grow single crystal of the title compound.

\subsection{Single crystal $X$-ray diffraction (XRD) study}

The microstructure of the grown L-asp doped ATOXAL crystal, such as unit vector lengths, interfacial angles, unit cell volume, space group and crystal system were estimated by subjecting the samples to single crystal X-ray diffraction using ENRAF NONIUS CAD4 X-ray diffractometer with $\mathrm{CuK} \alpha$ radiation source $(\lambda=1.5404 \AA)$. The single crystal XRD data shows that both pure and L-asp doped ATOXAL crystals belong to the triclinic crystallographic system $[4,5]$. The observed lattice parameters of the pure and L-asp doped ATOXAL crystals are compared and presented in Table 1. Since, the dopant concentration is $1 / 10$ of the atomic weight of the entire compound, the deviation in the unit cell parameters and volume is only marginal which reflects the incorporation of the L-asp molecule in the ATOXAL lattice.

\subsection{Fourier transform infrared (FT-IR) analysis}

Single crystal X-ray diffraction gives information about the physical measurements of structural phases demanding a high level of instrumental expertise [6]. Also, there is not much gain in structural resolution from the accuracy level
Table 2. Vibrational assignments of pure and L-asp doped ATOXAL.

\begin{tabular}{ccc}
\hline \multicolumn{2}{c}{ Wavenumber $\left[\mathrm{cm}^{-1}\right]$} & Assignment \\
L-asp doped & Pure \\
ATOXAL & ATOXAL & \\
\hline \hline 3437 & 3432 & $v(\mathrm{NH}), v(\mathrm{OH}) \mathrm{H}_{2} \mathrm{O}$ \\
3245 & & $v(\mathrm{OH})$ \\
1692,1432 & 1692 & $v_{\text {as }}(\mathrm{C}=\mathrm{O}), v_{\mathrm{s}}(\mathrm{C}=\mathrm{O}) \mathrm{COOH}$ \\
1432 & 1400 & $\mathrm{NH}_{4+}$ \\
1259 & 1251 & $v(\mathrm{C}-\mathrm{OH})$ \\
779 & 783 & $\rho\left(\mathrm{H}_{2} \mathrm{O}\right)$ \\
719 & 727 & $v(\mathrm{OH}) \mathrm{COOH}$ \\
& 567 & $v \mathrm{~L}\left(\mathrm{H}_{2} \mathrm{O}\right)$ \\
500 & & $\delta(\mathrm{NH})$ \\
482 & 481 & Rocking COO \\
\hline
\end{tabular}

of the obtained X-ray diffraction data values and this technique is rarely utilized [7]. Hence, the FT-IR technique was adopted to identify and confirm the existence of the functional groups present in the compound and thereby the chemical bonding and its molecular structure of the compound could be verified. The existing functional groups were analyzed in the spectral range of $4000 \mathrm{~cm}^{-1}$ to $400 \mathrm{~cm}^{-1}$ using the $\mathrm{KBr}$ pellet technique with the PerkinElmer Spectrum 1. Fig. 3 presents the FT-IR spectra of pure and L-asp doped ATOXAL. The vibrational frequencies of the pure and L-asp doped ATOXAL crystals are compared and presented in Table 2.

Absorption in the $4000 \mathrm{~cm}^{-1}$ to $400 \mathrm{~cm}^{-1}$ region is characterized by multiple fine structures on the lower wave number side of the band, similar to those observed in ATOXAL [5]. There is a broad strong absorption band between $3500 \mathrm{~cm}^{-1}$ and $2000 \mathrm{~cm}^{-1}$. It is due to the superimposed $\mathrm{OH}$ and $\mathrm{NH}$ stretching bands. The broad absorption peak at $3245 \mathrm{~cm}^{-1}$ is indicative of the intermolecular polymeric $\mathrm{OH}$ bond. The hydrogen bonding interaction between $\mathrm{NH}$ groups with the neighboring molecules in the crystal lattice contributes much to the peak broadening. The NH vibrational bond occurs at $3437 \mathrm{~cm}^{-1}$. The asymmetric and symmetric stretching vibrations at $1692 \mathrm{~cm}^{-1}$ 


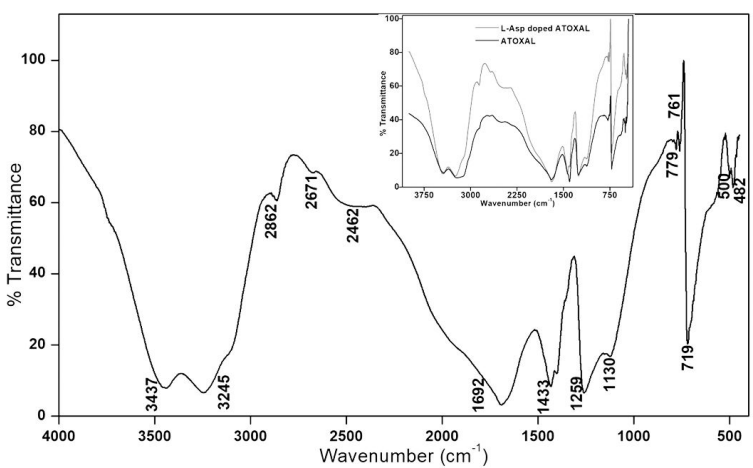

Fig. 3. FT-IR spectrum of L-asp doped ATOXAL; Inset - comparison of FT-IR spectra of pure and L-asp doped ATOXAL.

and $1432 \mathrm{~cm}^{-1}$ are due to $\mathrm{C}=\mathrm{O}$ in the carboxylic acid $\mathrm{COOH}$ group. The absorption peaks due to $\mathrm{NH}_{4}^{+}[8]$ and the vibration of the $\mathrm{C}-\mathrm{C}$ bond is overlapped with that of the $\mathrm{C}=\mathrm{O}$ symmetric stretching at $1432 \mathrm{~cm}^{-1}$. The vibration of $\mathrm{C}-\mathrm{OH}$ is visible at $1259 \mathrm{~cm}^{-1}$. The rocking of $\mathrm{H}_{2} \mathrm{O}$ and the deformation of $\mathrm{O}-\mathrm{C}=\mathrm{O}$ in the compound is observed at $779 \mathrm{~cm}^{-1}$. The peak at $719 \mathrm{~cm}^{-1}$ is due to the stretching vibration of the carboxylic acid and is more sensitive to the hydrogen bond interaction with amino acid dopants. The deformation due to the torsional oscillations of $\mathrm{NH}$ can be assigned to the peak at $500 \mathrm{~cm}^{-1}$. The rocking vibration of $\mathrm{O}-\mathrm{C}=\mathrm{O}$ is seen at $482 \mathrm{~cm}^{-1}$. Most of the peaks show a blue shift due the addition of the amino acid dopant.

\subsection{Linear optical studies}

\subsubsection{Diffused reflectance spectroscopic stud- ies}

When light is incident on a material it is reflected, transmitted, or absorbed. Conservation of energy dictates that the sum of the fractional amount of reflected, absorbed, transmitted and scattered light is unity. Scattering is a term used to describe diffuse reflectance (surface scatter) and diffuse transmittance (bulk scatter). When all incident and scattered light is assumed to be perfectly diffuse, and when only two opposite directions of light transport are considered, then the light intensity is assumed to vary along one axis only.

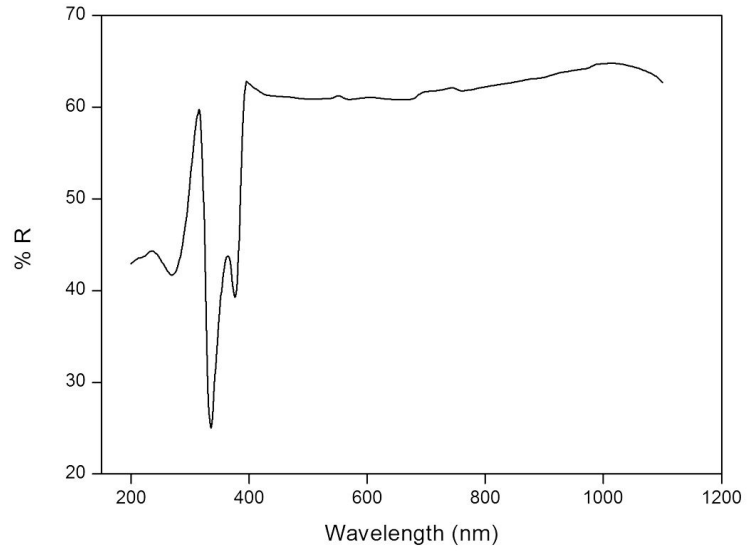

Fig. 4. Variation of diffused reflectance with wavelength in L-asp doped ATOXAL.

Kubelka et al. [9] gave a comprehensive formulation of the two-flux approximation of light diffusion. They presented a treatment with a clear aim towards practical methods of its measurement. Diffuse reflection is a light reflection that does not have strong directional properties and might occur also from a totally opaque, highly reflective but rough surface.

Diffused reflectance spectroscopy is a swift recce technique that locates the main regions of absorption and provides information on the energy, width, and intensity of absorption bands. The diffused reflectance spectrum of the title material is shown in Fig. 4.

The band gap $\left(\mathrm{E}_{\mathrm{g}}\right)$ of the L-asp doped ATOXAL material was calculated by adopting the KubelkaMunk theory. This theory gives the Kubelka-Munk remission function $\mathrm{f}(\mathrm{R})$ as [10]:

$$
f(R)=\frac{(1-R)^{2}}{2 R}=\frac{k}{s}
$$

where $\mathrm{R}, \mathrm{k}$ and s denote the reflectance, molar absorption and scattering coefficient of the sample, respectively. As the variation of $s$ with wavelength is slow, $\mathrm{f}(\mathrm{R})$ provides a good representation of the absorption spectrum of the crystal and is an equivalent of the absorption coefficient. From the plot of K-M of transformed reflectance spectrum, (f(R) $\mathrm{h} v)^{2}$ versus energy shown in Fig. 5, the optical band gap energy for L-asp doped ATOXAL was estimated by linear fit of the curve on the energy axis 
and it was found to be $3.731 \mathrm{eV}$. The lesser value of the band gap for L-asp doped ATOXAL than for pure ATOXAL (3.92 eV) [5] reveals that the band gap can be tuned to the required value by inclusion of dopants.

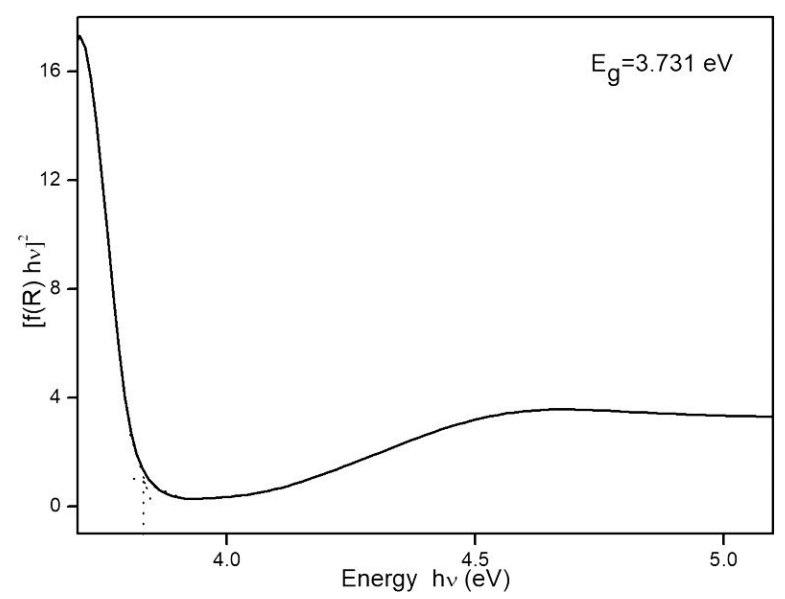

Fig. 5. K-M transformed reflectance spectra of L-asp doped ATOXAL.

\subsubsection{Absorbance/transmittance studies}

UV-Vis spectrophotometer (PerkinElmer Lamda 35) was used to record the optical absorption spectra of a $2 \mathrm{~mm}$ thick optically polished L-asp doped ATOXAL crystal in the wavelength range of $200 \mathrm{~nm}$ to $800 \mathrm{~nm}$, covering entire ultraviolet and visible region. A large transparent window in this region is vital for NLO characteristics. When an electron under an impact of an incident photon is excited to an intermediate state where it interacts with lattice vibrations and reaches a final state, the net result is the absorption of photons [11].

The suitability of the grown crystals for optical applications was confirmed. The grown crystal has a maximum absorption wavelength at $207 \mathrm{~nm}$. The plot of the absorption coefficient versus wavelength presented in Fig. 6 shows that the lower cutoff wavelength occurs at $343 \mathrm{~nm}$. Promotion of an electron between the ground and higher energy states from the $\sigma$ and $\pi$ orbitals is due to the absorbance of ultraviolet and visible light. The crystal has transparency with maximum transmittance being

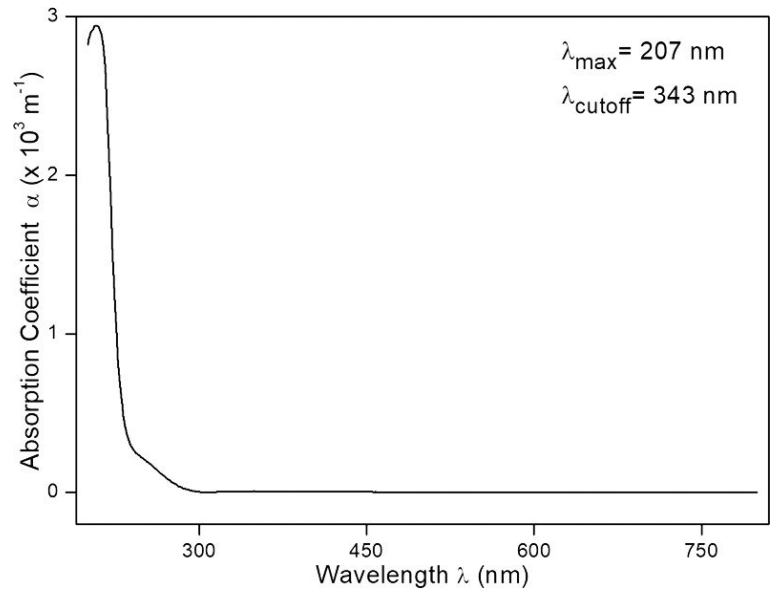

Fig. 6. Plot of absorption coefficient $\alpha$ vs. wavelength $\lambda$ for L-asp doped ATOXAL.

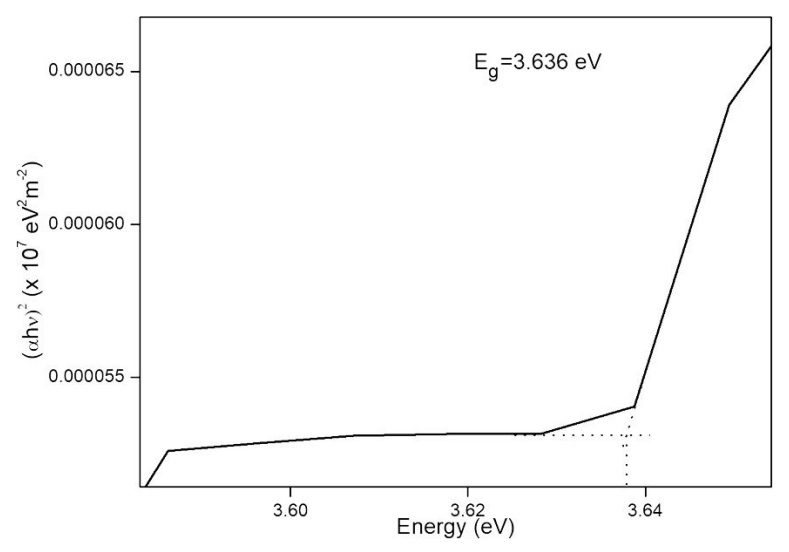

Fig. 7. Plot of $(\alpha h v)^{2}$ vs. energy hv for L-asp doped ATOXAL.

$99 \%$ in the entire visible region, which indicates that this crystal may be employed for applications in the entire visible and IR region.

Bardeen's model $[12,13]$ was adapted to determine the band gap and its nature. Assuming the photon energy to be small, the general equation for the band gap energy estimation is:

$$
(\alpha h v)^{p}=A\left(h v-E^{t}\right)
$$

where $\mathrm{A}$ is the disorder parameter, $\mathrm{E}^{\mathrm{t}}$ is the transition energy and $p$ denotes the type of optical transition whether allowed or forbidden. The transition can be direct for $\mathrm{E}^{\mathrm{t}}=\mathrm{E}_{\mathrm{g}}$ and indirect when $E^{t}=E_{g} \pm E_{p}$. Here, $E_{p}$ is the energy of the associated phonon. Curve fitting of the absorption coefficient in the absorption edge region yields $n=2$ 
for the allowed direct optical transition. From the plot of $(\alpha \mathrm{h} v)^{2}$ vs. energy hv presented in Fig. 7, the band gap was estimated to be $3.636 \mathrm{eV}$. From the absorbance, the transmittance was found using the relation:

$$
A=2-\log _{10} T
$$

When an electromagnetic wave of a wavelength $\lambda$ propagates through a material, the extinction coefficient indicates the measure of absorption loss. The relation between extinction coefficient and absorption coefficient is given by:

$$
K=\frac{\lambda \alpha}{4 \pi}
$$

where the absorption coefficient:

$$
\alpha=\frac{1}{t} \ln \left(\frac{1}{T}\right)
$$

Also, the reflectance $\mathrm{R}$ is related with the absorption coefficient as:

$$
R=\frac{1 \pm \sqrt{1-e^{-\alpha t}+e^{\alpha t}}}{1+e^{\alpha t}}
$$

The refractive index $\mathrm{n}$ can be calculated from reflectance data using the following relation:

$$
n=\frac{-(R+1) \pm \sqrt{3 R^{2}+10 R-3}}{2(R-1)}
$$

The refractive index of L-asp doped ATOXAL at the cutoff wavelength is 1.810 . The variation of extinction coefficient $\mathrm{K}$, refractive index $\mathrm{n}$ and reflectance $\mathrm{R}$ as a function of photon energy $\mathrm{h} v$, is shown in Fig. 8a to Fig. 8c. It is apparent from the plots that the extinction coefficient decays exponentially and reflectance remains constant and later both show a steep rise. The decrease of refractive index with an increase of energy is due to the inherent physical anisotropy in the material [1416]. The high percentage of transparency, low refractive index, low reflectance and extinction coefficient suggest the potential application of L-asp doped ATOXAL crystal as antireflection coating material for solar thermal devices $[17,18]$.

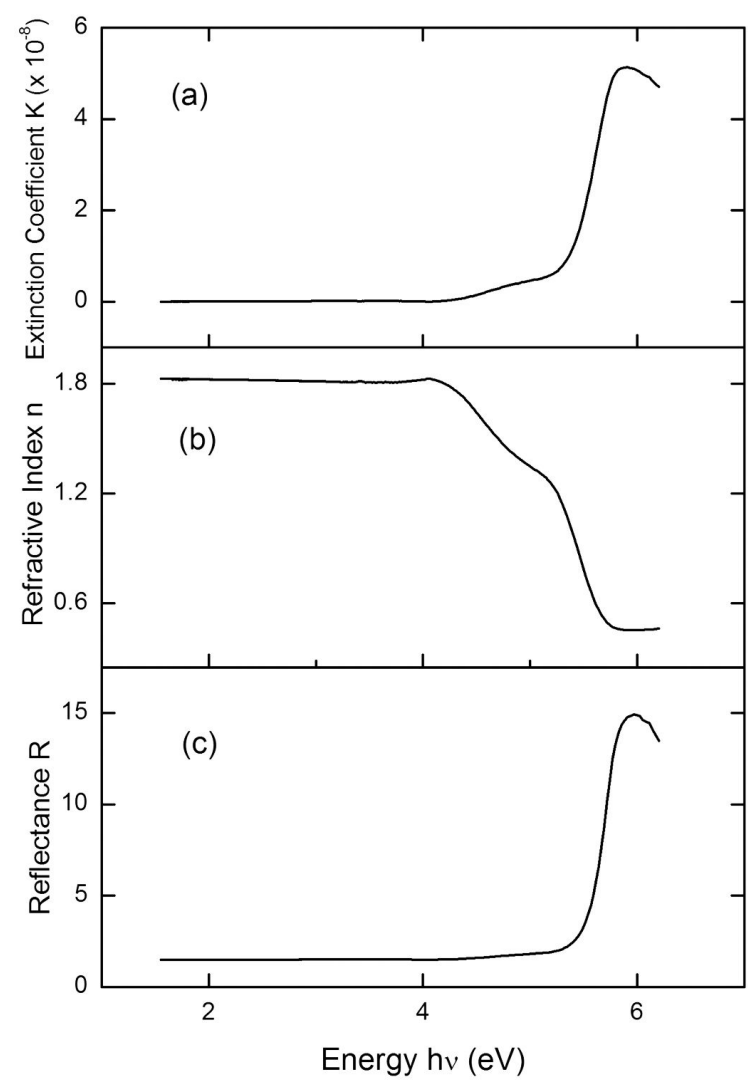

Fig. 8. Plots of (a) extinction coefficient K, (b) refractive index $n$ and (c) reflectance R vs. energy hv for L-asp doped ATOXAL.

Optical conductivity is one of the powerful tools for studying electronic states in materials. The optical conductivity $(\sigma)$ of the crystal was calculated using the following relation $[19,20]$ :

$$
\sigma=\frac{\alpha n c}{4 \pi}
$$

where $\mathrm{c}$ is the velocity of light. From the variation of the optical conductivity with energy plotted in Fig. 9, it is observed that the optical conductivity increases with energy and ATOXAL doping with L-asp enhanced the optical conductivity of the parent material.

The complex dielectric constant $\left(\epsilon=\epsilon_{\mathrm{r}}+\mathrm{i} \epsilon_{\mathrm{i}}\right)$ and the electric susceptibility $\chi_{c}$ were calculated using the relations by Gupta et al. [21]. The calculated values of the real $\epsilon_{\mathrm{r}}$ and imaginary $\epsilon_{\mathrm{i}}$ parts of dielectric constant and electric susceptibility $\chi_{c}$ 


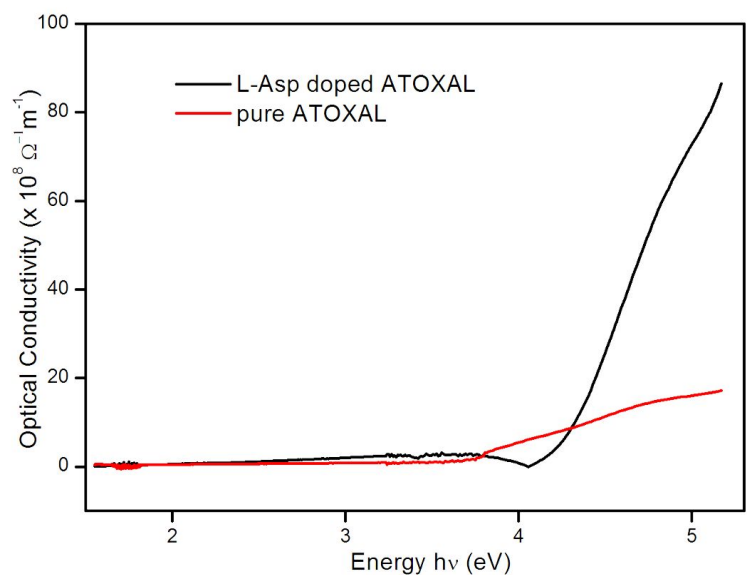

Fig. 9. Variation of optical conductivity with energy for pure and L-asp doped ATOXAL.

at the cutoff wavelength for pure and L-asp doped ATOXAL crystals are tabulated in Table 3.

\subsection{Microhardness study}

Assessment of mechanical properties, such as microhardness, offers useful information about a material strength and deformation characteristics. A measure of the material resistance to permanent deformation or damage, offered by the chemical forces during the motion of dislocations involving the displacement of atoms, is the hardness of the material. Hence, the crystal structure of the material is related to the hardness properties and corresponding studies are carried out by means of microhardness measurements. Mechanical properties of L-asp doped ATOXAL were determined and the related physical constants were calculated using a Vickers microhardness tester.

The quality of the specimen surface is very important for the indentation impression to be visible. A selected smooth and flat surface $\left(\begin{array}{lll}1 & 0 & \overline{1}\end{array}\right)$ of the grown crystal was subjected to microhardness study at room temperature with the load ranging from $1 \mathrm{~g}$ to $200 \mathrm{~g}$. The indentation time was $5 \mathrm{~s}$ for all the loads. The Vickers hardness number was calculated using the relation:

$$
H_{V}=1.8554\left(\frac{P}{d^{2}}\right)
$$

where $\mathrm{P}$ is the applied load in $\mathrm{kg}$ and $\mathrm{d}$ is the diagonal length of the indentation impression in $\mathrm{mm}$.
Hardness value is independent of the depth of the penetration as expected. Variation of the Vickers hardness value, $\mathrm{H}_{\mathrm{v}}$ with the load $\mathrm{P}$ is shown in Fig. 10.

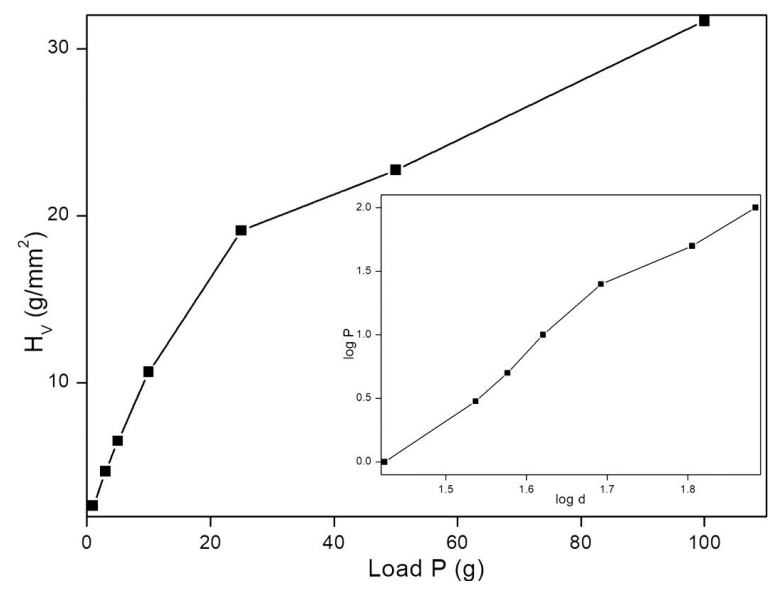

Fig. 10. Plot of hardness number $\mathrm{H}_{\mathrm{v}}$ vs. load $\mathrm{P}$ for L-asp doped ATOXAL; inset: plot of $\log$ P vs. $\log \mathrm{d}$ for L-asp doped ATOXAL.

The increase in hardness number with increasing load indicates the reverse indentation size effect (RISE). The RISE phenomenon is visible in crystals which readily undergo plastic deformation [22]. When a material is deformed by an indenter, dislocations are generated near the indentation site. The major contribution to the increase of hardness is attributed to the high stress required for homogenous nucleation of dislocations in the small indented dislocation-free region [23]. Meyer's law gives the relationship between the load and indentation size:

$$
P=k_{1} d^{n}
$$

where $\mathrm{k}_{1}$ is the standard hardness value and $\mathrm{n}$ is a constant for a particular material. Meyer number or work hardening coefficient $n$ is a measure of the indentation size effect (ISE) and is obtained from the plot of $\log \mathrm{P}$ versus $\log \mathrm{d}$, depicted in the inset of Fig. 10. The exponent $n$ is lower than 2 for a normal ISE behavior, and when $\mathrm{n}$ is higher than 2 , there is the RISE behavior [24]. $\mathrm{n}$ should be between 1 and 1.6 for hard materials and above 1.6 for the soft ones. The obtained value of $\mathrm{n}$ shows 
Table 3. Electrical parameters of pure and L-asp doped ATOXAL.

\begin{tabular}{|c|c|c|c|}
\hline \multirow[t]{2}{*}{ ATOXAL } & & constant & Electric susceptibility \\
\hline & \multicolumn{2}{|c|}{ Real $\epsilon_{\mathrm{r}}$ Imaginary $\epsilon_{\mathrm{i}} \times 10^{-7}$} & $\chi_{c}$ \\
\hline Pure & 1.744 & 9.97 & 0.139 \\
\hline L-asp doped & 3.276 & 6.44 & 0.261 \\
\hline
\end{tabular}

that L-asp doped ATOXAL crystal belongs to soft material category.

Various hardness parameters have been determined using Meyer's law, Wooster's empirical relation [25] and Kick's law. They were compared with pure ATOXAL and tabulated in Table 4.

The fracture stress applied under uniform loading is the fracture toughness $\mathrm{K}_{\mathrm{C}}$ and it is a measure of the material resistance to fracture or the toughness of the material. It is expressed by the relation:

$$
K_{C}=\frac{P}{\beta_{0} c^{3 / 2}}
$$

where $\beta_{0}$ is an indentation geometry dependent constant. The calculated $\mathrm{K}_{\mathrm{C}}$ for $\mathrm{L}$-asp doped ATOXAL with $\beta_{0}=7$ (Vicker's indenter) was $0.474 \mathrm{MNm}^{-3 / 2}$.

An important property that affects the mechanical behavior of a material is brittleness which gives an idea about the fracture induced in the material without any appreciable deformation. The value of the brittleness index $B_{i}$ is computed using the relation:

$$
B_{i}=\frac{H_{V}}{K_{C}}
$$

\subsection{Thermal analysis}

The thermal behavior was studied by thermogravimetric analysis (TGA) and differential thermal analysis (DTA) using a NETZSCH STA 409 $\mathrm{PC} / \mathrm{PG}$ thermal analyzer in the nitrogen atmosphere. The NETZSCH DSC 200F3 was used to study the phase transition that occurred in the crystal. The DSC curve in Fig. 11 shows thermal anomalies at $135.3{ }^{\circ} \mathrm{C}$ and $221.7{ }^{\circ} \mathrm{C}$ which are higher than those of pure ATOXAL [5]. The inset in Fig. 11 shows the DSC curve for pure ATOXAL crystal. The first endothermic peak at $135.3{ }^{\circ} \mathrm{C}$, where no significant weight loss over the course of the peak occurs, corresponds to the melting point of the sample. The TGA and DTG curves in Fig. 12 also support these observations. The inset of Fig. 12 represents the TG/DTA curves of pure ATOXAL. The first and second overlapped peaks in the DTG curve as well as the inflection in the TGA curve correspond to the dehydration of water molecules from the sample. From the analysis, it is concluded that the crystal decomposes before melting. The third peak and inflection in the DTA and TGA curves, respectively, relate to the melting of the sample. It is inferred from the thermal studies that the L-asp doped ATOXAL has a higher phase transition temperature than its parent compound [5].

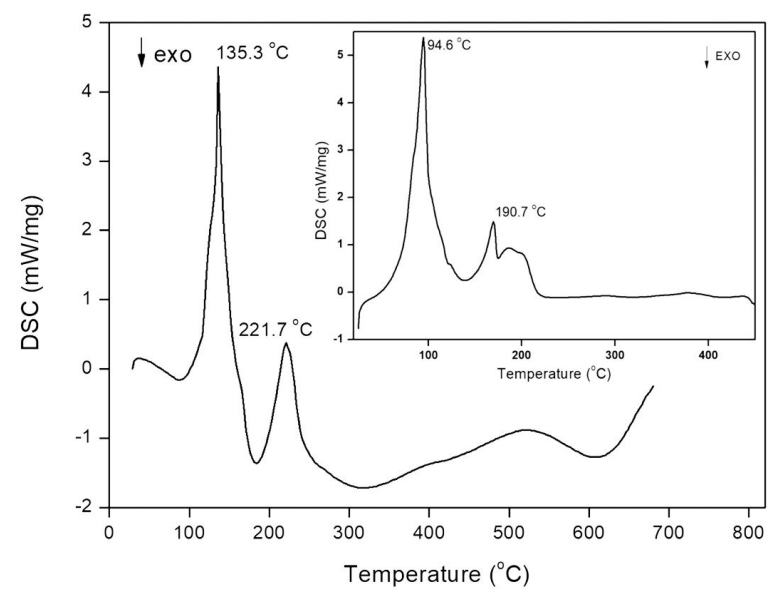

Fig. 11. DSC analysis of L-asp doped ATOXAL; (inset) DSC analysis of pure ATOXAL.

\subsection{Dielectric studies}

A silver paste was deposited on the both sides of the sample of L-asp doped ATOXAL crystal to make it behave like a parallel plate capacitor. Dielectric measurement was carried out using 
Table 4. Hardness parameters of L-asp doped ATOXAL.

\begin{tabular}{ccccccccc}
\hline ATOXAL & $\begin{array}{c}\mathrm{H}_{\mathrm{v}} \\
{\left[\mathrm{kg} / \mathrm{mm}^{2}\right]}\end{array}$ & $\mathrm{n}$ & $\begin{array}{c}\mathrm{k}_{1} \\
{\left[\times 10^{5} \mathrm{~kg} / \mathrm{m}\right]}\end{array}$ & $\begin{array}{c}\mathrm{k}_{2} \\
{\left[\times 10^{4}\right.} \\
\mathrm{kg} / \mathrm{m}]\end{array}$ & $\begin{array}{c}\mathrm{W} \\
{[\mathrm{g}]}\end{array}$ & $\begin{array}{c}\mathrm{c}_{11} \\
{\left[\times 10^{8}\right.} \\
\mathrm{Mpa}]\end{array}$ & $\begin{array}{c}\mathrm{x} \\
{[\mu \mathrm{m}]}\end{array}$ & $\begin{array}{c}\sigma_{\mathrm{y}} \\
{[\mathrm{Mpa}]}\end{array}$ \\
\hline \hline Pure & 39.98 & 2.917 & 4.017 & 3.844 & 16.07 & 635.83 & -0.0105 & 2.168 \\
L-asp doped & 19.109 & 3.112 & 2.835 & 1.795 & 14.176 & 174.65 & -0.005 & 0.491 \\
\hline
\end{tabular}

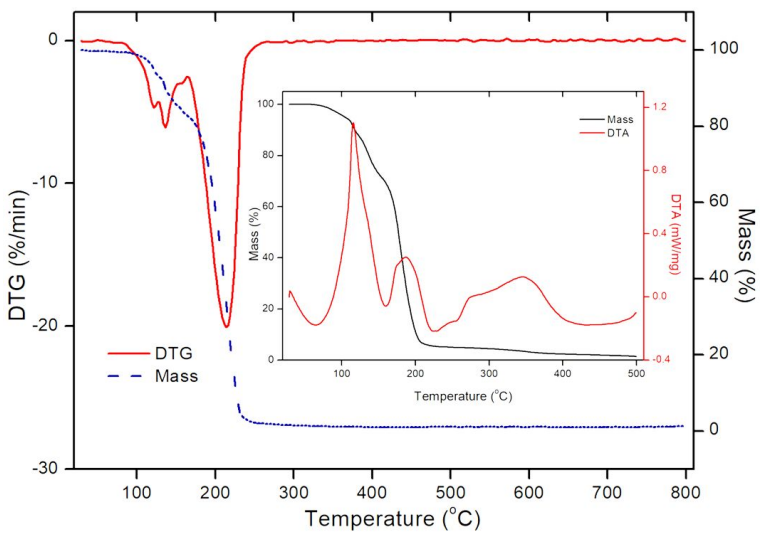

Fig. 12. TG/DTG analyses of L-asp doped ATOXAL; (inset) TG/DTA analyses of pure ATOXAL.

a HIOKI 3532-50 LCR HITESTER in the frequency range of $50 \mathrm{~Hz}$ to $1 \mathrm{MHz}$. The measurements were carried out in the temperature range of $125^{\circ} \mathrm{C}$ to $155^{\circ} \mathrm{C}$. The dielectric constant was calculated using:

$$
\varepsilon^{\prime}=\frac{C d}{\varepsilon_{0} A}
$$

where $\mathrm{C}$ is the capacitance, $\mathrm{d}$ is the thickness, $\mathrm{A}$ is the area and $\epsilon_{0}$ is the absolute permittivity of free space. Ferroelectric materials obey the Curie-Weiss law which relates the frequency dependent dielectric constant with temperature:

$$
\varepsilon^{\prime}(\omega)=B+\frac{C}{T-T_{C}}
$$

where $\mathrm{B}$ and $\mathrm{C}$ are temperature independent constants. The parameters $\mathrm{C}$ and $\mathrm{T}_{\mathrm{C}}$ are called the Curie-Weiss constant and the Curie temperature, respectively. Ignoring the first term due to the high magnitude of the second term in equation 14 , we get:

$$
\varepsilon^{\prime}(\omega)=\frac{C}{T-T_{C}}
$$

The frequency dependent dielectric constant in accordance with Debye's equation is:

$$
\varepsilon^{\prime}(\omega)=\varepsilon^{\prime}(\infty)+\left(\frac{\varepsilon^{\prime}(0)-\varepsilon^{\prime}(\infty)}{1+(\omega \tau)^{2}}\right)
$$

$\epsilon^{\prime}(\infty)$ is the dielectric constant at high frequency and equals to $n^{2}$, where $n$ is the optical refractive index. So, equation 16 can be rewritten in another form by putting $\epsilon^{\prime}(\infty)=\mathrm{n}^{2}$, then:

$$
\varepsilon^{\prime}(\omega)-n^{2}=\frac{\varepsilon^{\prime}(0)-n^{2}}{1+(\omega \tau)^{2}}
$$

For ferroelectric materials $\mathrm{n}^{2}$ is negligible with respect to $\epsilon^{\prime}(\omega)$ and $\epsilon^{\prime}(0)$, then:

$$
\varepsilon^{\prime}(\omega)=\frac{\varepsilon^{\prime}(0)}{1+(\omega \tau)^{2}}
$$

Debye's equation gives the frequency dependent dielectric loss as:

$$
\begin{aligned}
\varepsilon^{\prime \prime}(\omega) & =\left(\varepsilon^{\prime}(0)-\varepsilon^{\prime}(\omega)\right) \frac{\omega \tau}{1+(\omega \tau)^{2}} \\
& \approx \varepsilon^{\prime}(0) \frac{\omega \tau}{1+(\omega \tau)^{2}} \approx \varepsilon^{\prime}(\omega) \omega \tau
\end{aligned}
$$

using equation 18 , by substituting equation 15 in equation 19 , we obtain:

$$
\varepsilon^{\prime \prime}(\omega) \approx C \frac{\omega \tau}{T-T_{C}}
$$

then:

$$
\tau \approx \varepsilon^{\prime \prime}(\omega) \frac{T-T_{C}}{\omega C}
$$

Therefore, when $\mathrm{T} \rightarrow \mathrm{T}_{\mathrm{C}}$, then $\tau \rightarrow 0$ and equation 18 can be rewritten as $\epsilon_{\max }^{\prime}=\epsilon^{\prime}(0)$. Thus, the dielectric peak at the phase transition temperature in ferroelectric materials is $\epsilon^{\prime}(0)$ in the Debye's equation for the dielectric materials [26]. 


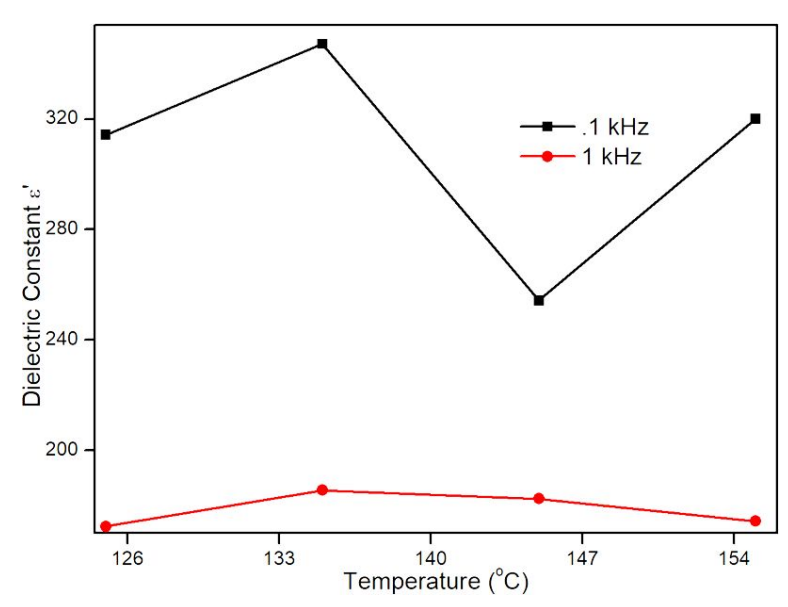

Fig. 13. Variation of dielectric constant $\epsilon^{\prime}$ with temperature in L-asp doped ATOXAL.

From the variation of dielectric constant with temperature plotted in Fig. 13 a thermal anomaly at $135{ }^{\circ} \mathrm{C}$ is observed. A similar anomaly was predicted in the thermal analysis discussed previously. An interaction between the dipole moments of the water molecules and the ionic charges occur, followed by the dehydration of the crystal just before the peak. Later, the ion-dipole interactions lead to a split between the ionic charges and water molecules. Thus, there is a space charge created by the thermally generated charge accumulation [27] at high temperatures. Moreover, a decrease in the dielectric constant is seen in L-asp doped ATOXAL.

A decrease in dielectric constant with an increase in frequency is observed and is assigned to the dominance of space charge polarization at low frequencies and the occurrence of electronic polarization alone at higher frequencies. This variation reveals the purity and perfection of the crystal. It is also observed that the dielectric loss $\tan \delta$ increases with temperature. This is due to a decrease in resistivity with an increase in temperature. The domain wall motion in the ferroelectric phase, domain shape change and mobility of domains are also attributed to the above said behavior.

The AC electrical conductivity $\sigma$ is calculated using the dielectric data and an empirical relation:

$$
\sigma=\omega \varepsilon^{\prime} \varepsilon_{0} \tan \delta
$$

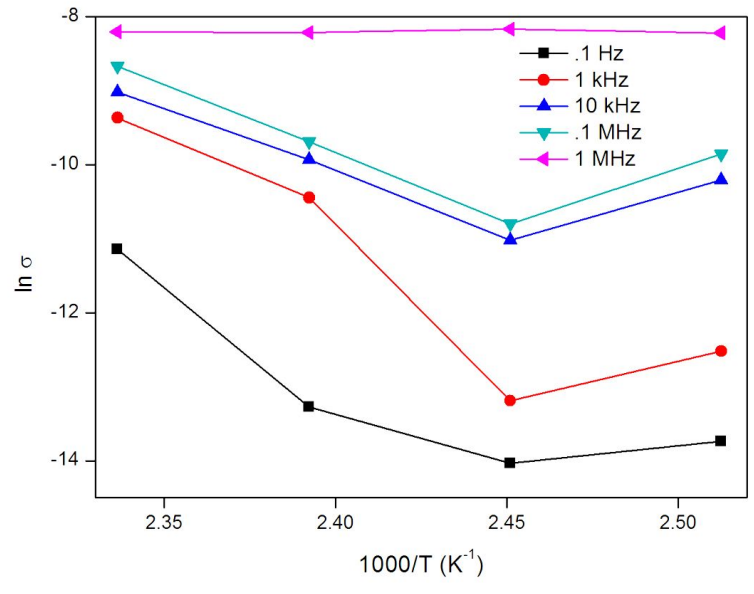

Fig. 14. Plot of $\ln \sigma$ vs. $\frac{1000}{T}$ for L-asp doped ATOXAL.

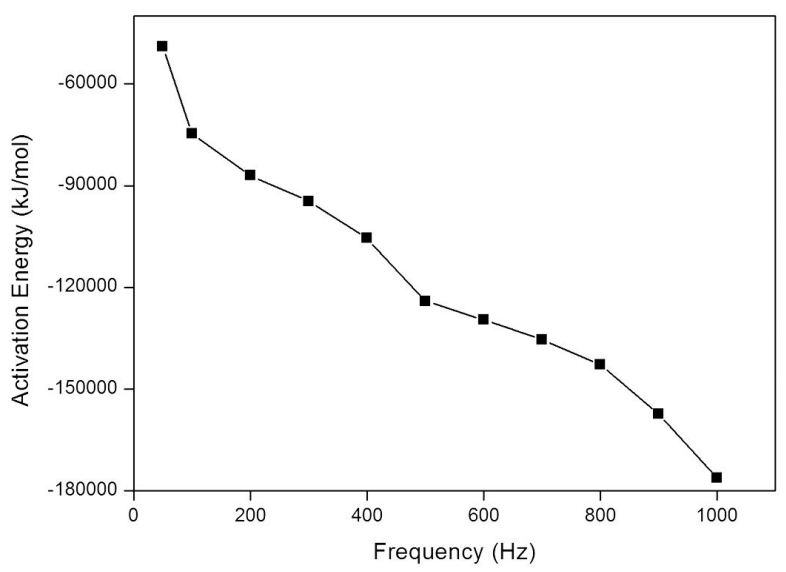

Fig. 15. Variation of activation energy with frequency in L-asp doped ATOXAL.

where $\epsilon_{\mathrm{o}}$ is the permittivity of free space and $\omega$ is the angular frequency.

The Arrhenius equation:

$$
\sigma=\sigma_{0} \exp \left(E_{a} / k T\right)
$$

is used to calculate the activation energy $E_{a}$. The plot representing the variation of $\ln \sigma$ with $1000 / \mathrm{T}$ is shown in Fig. 14. The variation of the activation energy with frequency is shown in Fig. 15.

The observed frequency dependent conductivity obeys Jonscher universal power law given by:

$$
\sigma(\omega)=\sigma_{0}+A \omega^{n}
$$




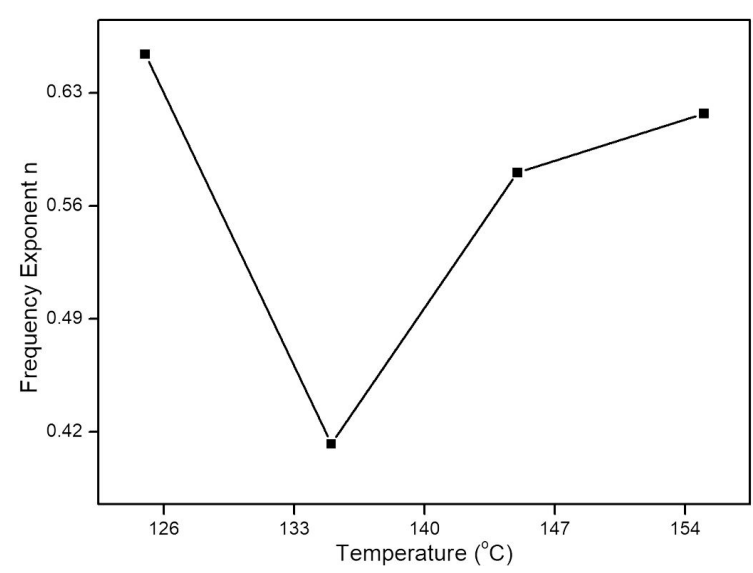

Fig. 16. Variation of the frequency exponent $n$ with temperature in L-asp doped ATOXAL.

where $\omega=2 \pi f$. The slope of $\ln \sigma$ with $\ln \omega$ gives the value of the frequency dependent exponent $\mathrm{n}$. From the variation of frequency exponent with temperature in Fig. 16 it is perceived that the exponent decreases with temperature up to the phase transition temperature and later increases. It is therefore inferred that many body interactions are the lowest at the phase transition temperature and higher at other temperatures.

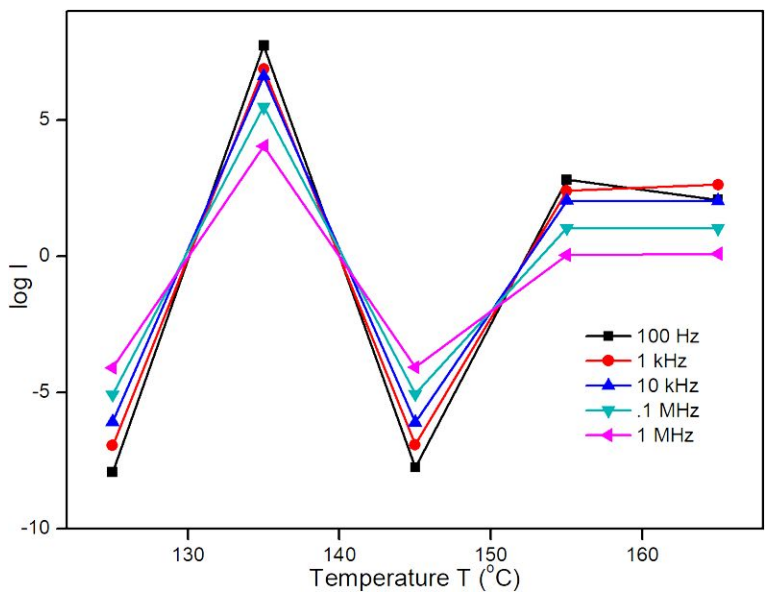

Fig. 17. Variation of pyroelectric current with temperature in L-asp doped ATOXAL.

The pyroelectric current measurement can be regarded as complementary to hysteresis loop measurement and applied to the Curie point transition. The variation of current with temperature for various frequencies obtained from the dielectric study is shown in Fig. 17, and it is seen that the current increases with temperature, and finally a critical temperature is reached above which pyroelectric current begins to decrease. The critical temperature is found to be $135^{\circ} \mathrm{C}$.

\subsection{Nonlinear optical study: Z-scan study}

The Z-scan technique is a highly accurate, very sensitive and yet a simple method backed by an elaborated and well understood theory to determine the nonlinear index of refraction $\mathrm{n}_{2}$ and nonlinear absorption coefficient $\beta$. The nonlinear refractive index is measured in the closed aperture mode while the open aperture mode measurement gives us information about the nonlinear absorption coefficient. The closed aperture, open aperture and ratio of closed to open normalized Z-scan measurements of L-asp doped ATOXAL with water as solvent at $64 \%$ transmittance is portrayed in Fig. 18.

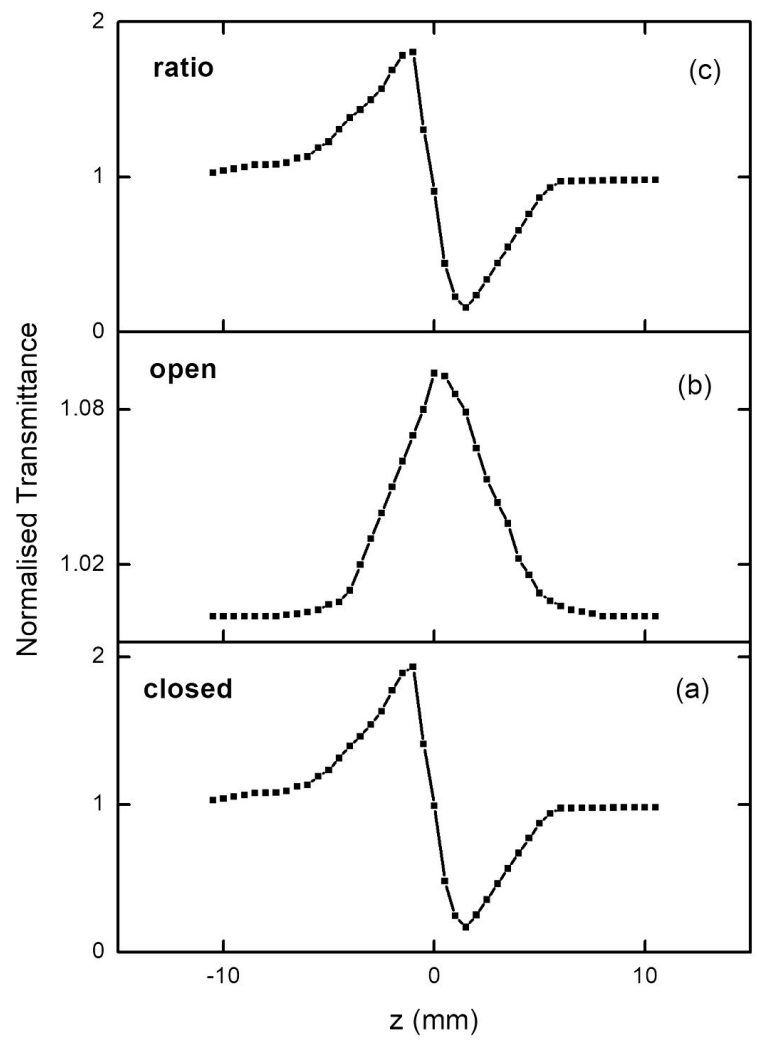

Fig. 18. Normalized transmittance curve from Z-scan measurements for (a) closed aperture (b) open aperture and (c) ratio of closed to open aperture for L-asp doped ATOXAL. 
Table 5. Nonlinear optical parameters of L-asp doped ATOXAL.

\begin{tabular}{ccccc}
\hline $\begin{array}{c}\mathrm{n}_{2} \\
{\left[\times 10^{-8} \mathrm{~cm}^{2} / \mathrm{W}\right]}\end{array}$ & $\begin{array}{c}\chi^{(3)} \\
{\left[\times 10^{-6} \mathrm{esu}\right]}\end{array}$ & $\begin{array}{c}\beta \\
{\left[\times 10^{-4} \mathrm{~cm} / \mathrm{W}\right]}\end{array}$ & $\begin{array}{c}\operatorname{Re} \chi^{(3)} \\
{\left[\times 10^{-6} \mathrm{esu}\right]}\end{array}$ & $\begin{array}{c}\operatorname{Im} \chi^{(3)} \\
{\left[\times 10^{-6} \mathrm{esu}\right]}\end{array}$ \\
\hline \hline 8.421 & 7.099 & 0.068 & 7.086 & 0.421 \\
\hline
\end{tabular}

The closed aperture Z-scan data indicate negative refraction nonlinearity, i.e. self-defocusing. This is caused by a local variation of refractive index with temperature. Night vision devices use this self-defocusing property. Separation of the effect of nonlinear refraction from that of the nonlinear absorption by dividing the closed aperture transmittance by the corresponding open aperture scan is an effective and approximate method to obtain $\mathrm{n}_{2}$. The nonlinear absorption coefficient $\beta$ can be determined from the open aperture $\mathrm{Z}$-scan data. The experimental values of $n_{2}$ and $\beta$ enable us the calculation of the real and imaginary parts of the third-order nonlinear optical susceptibility $\chi^{(3)}$ and eventually the third-order nonlinear optical susceptibility $\chi^{(3)}$. The nonlinear parameters were calculated [28] and the obtained values are tabulated in Table 5 .

\subsection{P-E loop trace}

Fig. 19 shows the polarization vs. electric field (P-E) hysteresis loops of L-asp doped ATOXAL for various poling fields at room temperature. The sample exhibits well defined ferroelectric loops. The values of remnant polarization $\left(\mathrm{P}_{\mathrm{r}}\right)$, saturation polarization $\left(\mathrm{P}_{\mathrm{s}}\right)$, coercive field $\left(\mathrm{E}_{\mathrm{c}}\right)$ and the area of the loop for some poling fields are listed in Table 6. It can be seen that the $\mathrm{P}_{\mathrm{s}}, \mathrm{P}_{\mathrm{r}}, \mathrm{E}_{\mathrm{c}}$ and the loop area decrease systematically with a decrease in the poling field. Also, when the residual and tensile stresses decrease, a reduction in the aforesaid parameters occurs. The shape of the loops reveals the good strain free quality of the crystal. The room temperature electric field dependences of the remnant polarizations and coercive fields measured by ferroelectric hysteresis loops show that the remnant polarization increases with increasing the electric field, reaches to approximately $746 \mathrm{pC} / \mathrm{cm}^{2}$ at $2 \mathrm{kV} / \mathrm{cm}$ and does not show the tendency of saturation. Hence, polarization increases and leads to a 'hardening' of the ferroelectric material. The ferroelectric hysteresis loops have a pinched-like shape which is common when domain walls are pinned by defects. Most of these defects are acceptor-oxygen-vacancy dipoles. Similar but intentionally introduced defects are also known to lead to pinched PE loops. Thus, the presence of the amino acid dopant has introduced defects into the crystal structure of ATOXAL which led to the pinching effect. The loop area shows an increase with a widening of the pinch as the poling field increases. This could be attributed to the coexistence of the disordered and ordered dipoles around the vicinity of the dopant induced defect. An important characteristic of a ferroelectric material hysteresis curve is the squareness of the loop given by:

$$
R_{s q}=\frac{P_{r}}{P_{s}}+\frac{P_{1.1 E_{C}}}{P_{r}}
$$

where $R_{s q}$ and $P_{1.1 \mathrm{Ec}}$ represent squareness of the P-E loop and the polarization under the external field of $1.1 \mathrm{E}_{\mathrm{c}}$, respectively. The $\mathrm{R}_{\mathrm{sq}}$ value was determined to be 0.552 . Hence, we observe the existence of a strong relationship between the shape of the hysteresis loop, the nature of the material as well as the applied electric field during poling.

\section{Conclusions}

Single crystals of L-asp doped ATOXAL were grown by the conventional slow evaporation solution growth method. The FT-IR spectral analysis and single crystal X-ray diffraction proved the inclusion of L-asparagine in the ATOXAL crystal structure. A blue shift is visible in the FTIR spectrum. From the DRS and UV-Vis spectroscopic studies it is observed that L-asp doped ATOXAL has a relatively lesser optical band gap of $3.636 \mathrm{eV}$. Other optical constants, such as the refractive index, dielectric constants and electric 

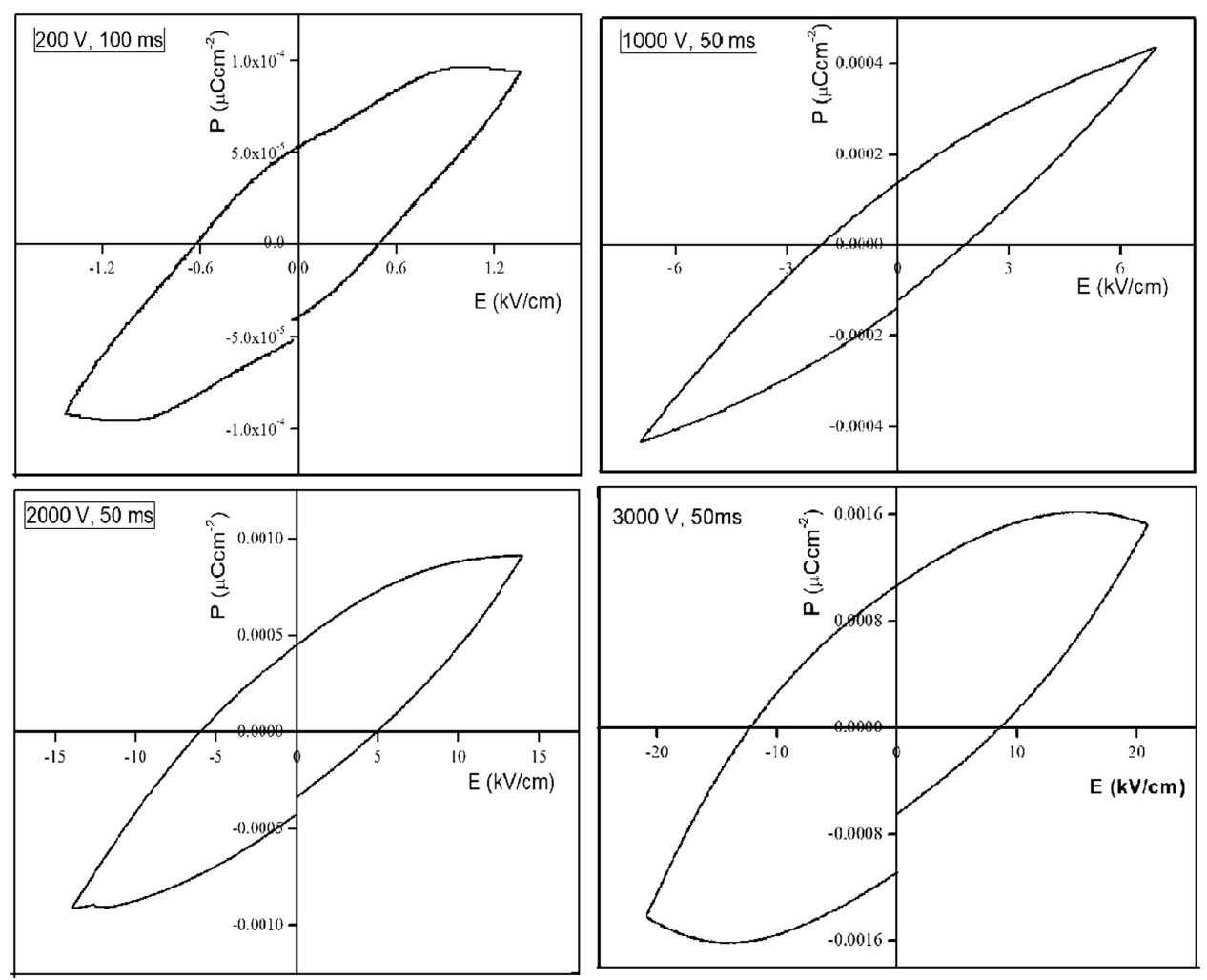

Fig. 19. P-E hysteresis loops for L-asp doped ATOXAL under various poling field strengths.

Table 6. Ferroelectric data of L-asp doped ATOXAL.

\begin{tabular}{ccccc}
\hline Poling field $[\mathrm{V}]$ & 3000 & 2000 & 1000 & 200 \\
\hline \hline Saturation polarization $\mathrm{P}_{\mathrm{s}}\left[\mathrm{pC} / \mathrm{cm}^{2}\right]$ & 1520 & 908 & 435 & 94 \\
Remnant polarization $\mathrm{P}_{\mathrm{r}}\left[\mathrm{pC} / \mathrm{cm}^{2}\right]$ & 1060 & 450 & 137 & 53 \\
Coercive field $\mathrm{E}_{\mathrm{c}}[\mathrm{kV} / \mathrm{cm}]$ & 1230 & 706 & 258 & 70 \\
Capacitance $\mathrm{C}[\mathrm{pF}]$ & 12.7 & 11.3 & 10.9 & 11.7 \\
PE loop area $\left[\mu \mathrm{C} / \mathrm{cm}^{2}-\mathrm{V}\right]$ & 8.21 & 2.31 & 0.351 & 0.0263 \\
\hline
\end{tabular}

susceptibility were also found. Vickers microhardness test was carried out and hardness parameters were calculated. The Meyer's number value categorized the sample to be a soft material. It is interesting to note that the incorporation of dopant has slightly decreased the hardness of the parent material. Thermal anomalies on the higher scale compared to ATOXAL were observed in the DSC and TG/DTA curves and subsequent dielectric studies also revealed such anomalies suggesting the possibility of ferroelectric nature for the title material. The material could be a potential third order nonlinear material due to its centrosymmetric nature. The PE hysteresis loops show a pinched effect due to the inclusion of the dopant induced defect. Thus, L-asp ATOXAL is an unconventional third order nonlinear optical and ferroelectric system, possessing a centrosymmetric structure with the development of weak electric polarization above the dielectric transition of around $135.3{ }^{\circ} \mathrm{C}$. Although the global crystallographic structure is triclinic and centrosymmetric $(\mathrm{P} \overline{1})$, in the ferroelectric-like state it is locally non-centrosymmetric due to presence of defects 
induced by the hydrogen bonds of the dopant. Hence, in conclusion, a local displacement might have occurred and could explain the weak ferroelectric like polarization. A clear understanding of this 'local non-centrosymmetry' will be of great significance to explain the unusual properties of such multifunctional materials.

\section{References}

[1] Lines M.E., Glass A.M., Principles and Applications of ferroelectric and related material, Oxford University Press, New York, 1977.

[2] Dauber M., Rabe K.M., Scott J., Rev. Mod. Phys., 77 (2005), 1083.

[3] Twieg R., Azema A., Jain K., Cheng Y.Y., Chem. Phys. Lett., 92 (1982), 208.

[4] Currie M., Speakman J.C., Curry N.A., J. Chem. Soc. A, (1967), 1862.

[5] Jerusha E., Kumar R.I.S., Kirupavathy S.S., Gopalakrishnan R., Optik, 127 (2016), 3896.

[6] Amirkhanyan Z.G., Remedios C.M., MASCARENHaS Y.P., MorelhaO S.L., J. Appl. Cryst., 47 (2014), 160.

[7] Soares A.S., Caspar D.L., Weckert E., HerouX A., Hoelzer K., Schroer K., Zellner J., SChneider D., Nolan W., SweEt R.M., Acta Cryst. Sec. D: Bio. Cryst., 59 (2003), 1716.

[8] Colthup N.B., J. Opt. Soc. Am., 40 (1950), 397.

[9] Kubelka P., J. Opt. Soc. Am., 44 (1954), 330.

[10] Burgeth G., Kisch H., Coord. Chem. Rev., 230 (2002), 41.

[11] Mersch M., Buse K., Sauf W., Hesse H., Kratzig E., Phys. Status Solidi, 140 (1993), 273.

[12] Bardeen J., Blatt F.J., Hall L.H., Proceedings of the Photoconductivity conference, Atlantic City, Wiley, New York, (1956), 146.
[13] Schmcisser D., Rager A., Thonke K., PilkuhN M., Frohlich D., GaAuglitz G., Schafer M., Oelkrug D., Synth. Met., 41 (1991), 1457.

[14] Fang Z., Lin J., LiU R., LiU P., Li Y., Huang X., Ding K., Ning L., Zhang Y., Cryst. Eng. Commun., 16 (2014), 10569.

[15] Senthil K., Kalainathan S., Kumar A.R., ArAVINDAN P.G., RSC Adv., 4 (2014), 56112.

[16] Dalal J., Sinha N., Kumar B., Opt. Mater., 37 (2014), 457.

[17] Anis M., Shirsat M.D., Hussaini S.S., Joshi B., Muley G.G., J. Mat. Sci. Tech., 32 (2016), 62.

[18] Girisun T.C.S., Dhanuskodi S., Cryst. Res. Technol., 44 (2009), 1297.

[19] Arumanayagam T., Murugakoothan P., J. Min. Mater. Char. Eng., 13 (2011), 1225.

[20] Ugwu E.I., Olayinka A.S., Olabode F.I., J. Eng. Appl. Sci., 4 (2009), 126.

[21] Gupta V., Mansingh A., J. Appl. Phys., 80 (1996), 1063.

[22] Li H., Han Y.H., Bradt R.C., J. Mater. Sci., 29 (1994), 5641-5645.

[23] Kunjomana A.G., Chandrasekharan K.A., Cryst. Res. Technol., 40 (2005), 782.

[24] Sangwal K., Mater. Chem. Phys., 63 (2000), 145.

[25] Wooster W.A., Rep. Prog. Phys., 16 (1953), 62.

[26] Krishnan S., RAJ C.J., Robert R., RAMAnAnd A., DAS S.J., Soli. Stat. Elect., 52 (2008), 1157.

[27] Vonhippel A.R., Materials and Applications, New York, (1965), 79.

[28] Sheik-Bahae M., SAid A.A., Wei T., Hagan D.J., Stryland E.W.V., IEEE J. Quant. Elect., 26 (1990), 760.

Received 2017-12-06

Accepted 2018-04-23 